\title{
Pipe material effect on water network leak detection using a pressure residual vector method
}

\begin{abstract}
Municipal authorities face many challenges in maintaining an effective water distribution service (WDS) because of aging infrastructure and financial limitations. Effective leak detection methods minimize the cost of pipe inspection and reduce the cost of leaks caused by deterioration in the pipe. The pressure residual procedure for leakage detection was performed by comparing the pressure data of certain district meter area (DMA) inner nodes with an estimation using the simulation of a mathematical network model. The effects of leakage in WDSs indicate that the pressure exponent is dependent on the geometry of the orifice, which is considered a constant factor in the applied method. This paper investigates the influence of pipe material in the pressure residual method and determines the element change in the sensitivity matrix. For accuracy, the geographic information technology (spatial data) was used to calculate the connection density around the exact nodes in the network model. The influence of the external load (soil movement and excavation work) on the metallic pipe material is beyond the scope of this paper, because leaks caused by these factors are often obvious and are accompanied by a huge pressure drop inside the distribution network.
\end{abstract}

Keyword: Water pipelines; Spatial data; Pipelines; Pipe leakage 